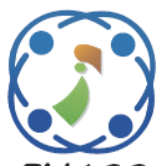

\title{
Airborne Computer System Path-Tracking Based Multi-PID-PSO Controller Design
}

\author{
Mohammed Najm Abdullah ${ }^{1 *}$ \\ Khulood Eskander Dagher ${ }^{2}$ \\ ${ }^{1}$ Computer Engineering Department, University of Technology, Baghdad, Iraq \\ ${ }^{2}$ Al-Khwarizmi College of Engineering, University of Baghdad, Baghdad, Iraq \\ * Corresponding author's Email: 120002@uotechnology.edu.iq
}

\begin{abstract}
A model avionics system is a type of electronic system and equipment specifically designed for use in airborne computers. This article proposes an improvement of the output performance of Unmanned Aerial Vehicle (UAV) system based on multi-PID-controller with on-line swarm optimization algorithm. The main goal of this research is to design six PID controllers to control the high nonlinear UAV quadcopter system by using an on-line Particle Swarm Optimization (PSO) algorithm that uses to learn and tune the eighteen control gain parameters based on multi-objective function. The task of the proposed on-line multi-PID-PSO feedback path-tracking controller is to obtain precisely and quickly the robust rotation speed of rotors which are used to control the attitude and altitude of the UAV quadcopter system. The results of the proposed strategy show that the on-line multi-PID-PSO controllers are accurate in terms of the UAV quadcopter take-off and follows the Spiral and Cyclone desired paths quickly through fast obtaining of the multi-controller's parameters and smooth rotation speeds actions generating for UAV system with a minimum number of multi-cost function evolutions that minimized the tracking translation location error around \pm 5 $\mathrm{cm}$ and the overshoot of altitude did not exceed $1 \mathrm{~cm}$. Finally, we confirm the effectiveness of the simulation results of the proposed controller through comparison of other types of controller simulation results.
\end{abstract}

Keywords: Unmanned aerial vehicle, Quadrotor, Multi-PID controller, Particle swarm optimization algorithm, Online tuning, Multi-objective function.

\section{Introduction}

In the past two decades, many researchers have been interested in Unmanned Aerial Vehicles (UAV)-based airborne computing, quadcopter or drone quad copter which have been applied in various domains such as military, industrial, and commercial applications. As well as UAV quadcopter is useful in different fields such as monitoring system and inspection system in dangerous area although it has different sizes and shapes [1]. To solve the control problem for the UAV quadrotor path tracking system, recently, many works have been reported in the literature for various types of mathematical models, controllers design and tuning control methodologies that are used to try for solving the problem of the UAV quadcopter stabilization in a desired location and orientation by controlling the attitude and altitude of UAV quadcopter. Some of the researchers have focused on controller design based on the mathematical model of the physical nonlinear unmanned aerial vehicle system such as: the hybrid intelligent controller that consisted of the robust adaptive neuro-fuzzy inference system and PSO algorithm which was proposed in [2] that are used for trajectory tracking of unmanned aerial vehicle, but the controller has only the ability for movement of the $\mathrm{UAV}$ in a $2 \mathrm{D}$ vertical plane. In the work [3], the decoupling fuzzy controller based on the NewtonEuler method of the mathematical kinematic and dynamic quadrotor UAV model are used to control the desired position of the quadrotor system, but the issue is taken by the linearization model of UAV. The fuzzy PID control algorithm is applied to dynamic quad-rotor UAV model to achieve robust attitude flight that are used in [4] so the weakness is not designed for the altitude controller for UAV model. 
On the other hand, in the paper [5] a PID controller with a nature-inspired algorithm is proposed to achieve good quadrotor performance for tracking the desired path but the drawback is the parameters of the PID controller are fixed off-line and not updated by on-line method. Also, multi-input-multi-output PID controller is presented in [6] by using linear quadratic regulator-gaussian for attitude and altitude control of the quadrotor but the drawback is taken from the linearization model of quadrotor model. Tuning PID controller by using two different methods; the first is conventional Zeigler-Nichols method and the second is modern heuristics approach of PSO method are illustrated in [7] to identify the best PID controller parameters for attitude stabilization of the quadrotor model but the disadvantage is not designed of the altitude controller for quadrotor model. The autotuner of cascade PID controller is discussed in [8] for quadrotor UAV model based on the desired gain and phase margins to determine the response speed than primary control it but the drawback is taken to the linearization model of quadrotor model. Tuning attitude PD controller by using two methods; ZeiglerNichols method and genetic algorithm are presented in [9] to find the best value of the PD controller parameters for attitude quadrotor helicopter model but the drawback is taken by the linearization model of quadrotor model. In addition, an intelligent adaptive type-2 fuzzy sliding mode control with PID sliding surface is proposed in [10] for controlling the 6 DOF quadrotor aircraft but the drawback is the parameters of the PID controller are fixed not updated. In the work [11] the Lyapunov gain Scheduled PID controller design for the model vertical flying drone quadrotor type with conventional PID and adaptive PID controllers. The tracking control of UAV quadrotor is developed in [12] where two controllers are used, traditional sliding mode control law and integral with gain scheduled sliding mode control in order to eliminate the disturbance error that effect on the UAV flight. The robustness and the effectiveness of the optimal model free backstepping controller with cuckoo search algorithm for quadrotor helicopter is proposed in [13] to achieve the global asymptotical stability of the closed loop control quadrotor helicopter based on the Lyapunov method but the issue is not compared with other optimization algorithms. Also, in the work [14] a backstepping controller is used for quadrotor based on NewtonEuler formulation to derive the reference attitude angles and to obtain asymptotical stabilized but the weakness is the parameters of the nonlinear controller did not update on-line. Moreover, model predictive of nonlinear controller for UAV is discussed in [15] for tracking desired trajectory and constrained control of UAV quadrotor system but the drawback is taken by the linearization model of quadrotor model. In [16] adaptive model predictive control is proposed to quadrotor altitude control that utilizing a dynamic matrix control for computing control action to improve quadrotor control and tracking. So, another type of control strategy for UAV system is a neural network controller as discussed in [17-19] where the outputs of these controllers are determined by the best values of position and speed of the quadrotor and for steering rotation speed of propellers to get the best quadrotor's flight of the desired path. The problem definition of this work is still to be addressed which the highly coupled between the inputs and the outputs states of the UAV quadrotor and the nonlinear dynamic behaviour with under-actuated system in order to quickly fly the UAV and to accurate and precise track of the desired path in terms of minimum position error, without oscillation and minimum overshoot in the altitude of quadrotor system. Therefore, the motivation of this work is taken from $[1,10,11,20]$. The summarized contribution of this paper is to solve the problem statement through a swift with best values of four rotor speeds control actions that are generated using numerical simulation based on the proposed on-line multi-PID-PSO control algorithm with proposed multi-objective function. Therefore, the effectiveness of the proposed technique leads to track and stabilization of the drone quadrotor in the strongly dynamics desired locations and orientations with minimum tracking error through control of the altitude and attitude of quadrotor system. This article is organized as follows: Section 2 explains the mathematical drone dynamics model. Section 3 illustrates the proposed multi-PID-PSO control strategy based on multi-objective function. In Section 4 , numerical simulation results are demonstrated by the effectiveness and of the performance of the proposed controller. Finally, the conclusions for the proposed control strategy are given in Section 5.

\section{UAV quadrotor drone dynamics model}

In general, the UAV quadrotor motion is relating of the center of mass movement (x,y and $\mathrm{z}$ ) of the altitude and movement around the center of mass $(\varphi$, $\theta$, and $\psi$ ) of the rotation. Therefore, $6 \mathrm{DOF}$ are needed to describe the UAV quadrotor motion in the space as shown in Fig. 1. The UAV quadrotor has four rotor motors to control the attitude and altitude of UAV quadrotor system, where the lift of the UAV quadrotor along the z-axis by varying four rotor speeds, to move the quadrotor along the $\mathrm{x}$-axis by 


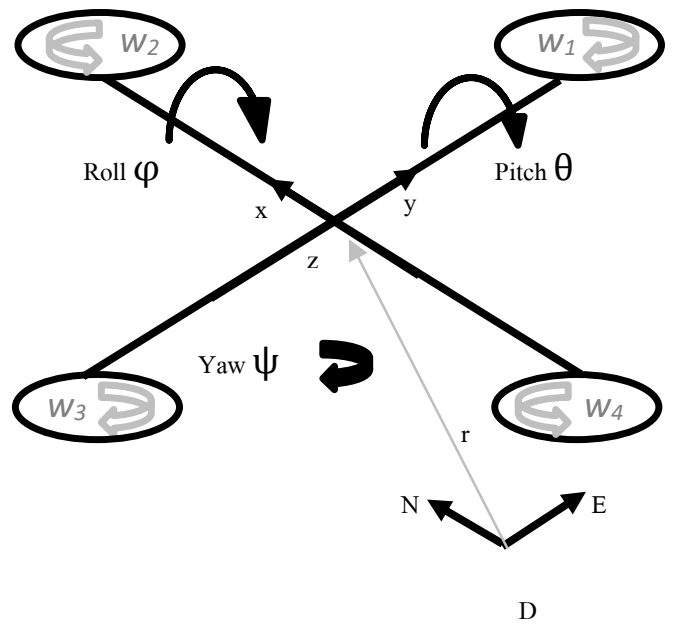

Figure. 1 The platform of drone quadrotor motion

varying the speed of rotors (w1) and (w3), so the pitch angle $(\theta)$ of the quadrotor is changing.

To move the quadrotor along the $y$-axis by varying the speed of rotors $\left(\mathrm{w}_{2}\right)$ and $\left(\mathrm{w}_{4}\right)$, so the roll angle $(\varphi)$ of the quadrotor is changing. To create yaw angle $(\psi)$ and rotate the UAV quadcopter w.r.t. z-axis, the speeds of rotors $\left(\mathrm{w}_{1}\right)$ and $\left(\mathrm{w}_{3}\right)$ are increased while the speeds of rotors $\left(\mathrm{w}_{2}\right)$ and $\left(\mathrm{w}_{4}\right)$ are decreased. The UAV quadcopter dynamic equation model are taken from $[5,10]$ with consideration of air drag as follows:

$$
\begin{gathered}
\ddot{x}=\frac{b}{M}(C \varphi S \theta C \psi+S \varphi S \psi) \times\left(w_{1}^{2}+w_{2}^{2}\right. \\
\left.+w_{3}^{2}+w_{4}^{2}\right) \\
\ddot{y}=\frac{b}{M}(C \varphi S \theta C \psi-S \varphi C \psi) \times\left(w_{1}^{2}+w_{2}^{2}\right. \\
\left.+w_{3}^{2}+w_{4}^{2}\right) \\
\ddot{z}=\frac{b}{M}(C \varphi C \theta)\left(w_{1}^{2}+w_{2}^{2}+w_{3}^{2}+w_{4}^{2}\right) \\
-g \\
\ddot{\varphi}=\frac{\left(I_{y}-I_{z}\right)}{I_{x}} \dot{\theta} \dot{\psi}-\frac{J_{r} \Omega_{r}}{I_{x}} \dot{\theta}+\frac{l b}{I_{x}}\left(w_{4}^{2}\right. \\
\left.-w_{2}^{2}\right) \\
\ddot{\theta}=\frac{\left(I_{z}-I_{x}\right)}{I_{y}} \dot{\varphi} \dot{\psi}+\frac{J_{r} \Omega_{r}}{I_{y}} \dot{\varphi}+\frac{l b}{I_{y}}\left(w_{3}^{2}\right. \\
\left.-w_{1}^{2}\right) \\
\ddot{\psi}=\frac{\left(I_{x}-I_{y}\right)}{I_{z}} \dot{\theta} \dot{\varphi}+\frac{d}{I_{z}}\left(w_{1}^{2}-w_{2}^{2}+w_{3}^{2}\right. \\
\left.-w_{4}^{2}\right)
\end{gathered}
$$

Table 1. Definition of drone quadrotor's parameters with values and units [5].

\begin{tabular}{|c|c|c|c|}
\hline Name & Parameter & Value & Unit \\
\hline $\begin{array}{c}\text { Quadcopter } \\
\text { Mass }\end{array}$ & $M$ & 0.65 & $\mathrm{Kg}$ \\
\hline $\begin{array}{c}\text { Quadcopter Arm } \\
\text { Length }\end{array}$ & $L$ & 0.23 & $\mathrm{M}$ \\
\hline $\begin{array}{c}\text { Thrust } \\
\text { Coefficient }\end{array}$ & $B$ & $\begin{array}{l}3.13 \\
\times 10^{-5}\end{array}$ & $\mathrm{~N} \cdot \mathrm{sec}^{2}$ \\
\hline Drag Coefficient & $d$ & $\begin{array}{l}7.5 \\
\times 10^{-7}\end{array}$ & $\mathrm{~N} \cdot \mathrm{m}^{-\mathrm{sec}^{2}}$ \\
\hline Inertia on x-axis & $I x$ & $\begin{array}{l}7.5 \\
\times 10^{-3}\end{array}$ & $\mathrm{~kg} \mathrm{~m}{ }^{2}$ \\
\hline Inertia on y-axis & $I_{y}$ & $\begin{array}{l}7.5 \\
\times 10^{-3}\end{array}$ & $\mathrm{~kg} \mathrm{~m}{ }^{2}$ \\
\hline Inertia on z-axis & $I_{z}$ & $\begin{array}{l}1.3 \\
\times 10^{-2}\end{array}$ & $\mathrm{~kg} \mathrm{~m}{ }^{2}$ \\
\hline Rotor Inertia & $J_{r}$ & $6 \times 10^{-5}$ & $\mathrm{~kg} \mathrm{~m}{ }^{2}$ \\
\hline Gravity constant & $g$ & 9.81 & $\mathrm{~m} / \mathrm{sec}^{2}$ \\
\hline $\begin{array}{c}\text { Rotational } \\
\text { Velocities }\end{array}$ & $\Omega$ & - & $\mathrm{rad} / \mathrm{sec}^{2}$ \\
\hline
\end{tabular}

Where, $(\ddot{x}, \ddot{y}, \ddot{z})$ and $(\dot{x}, \dot{y}, \dot{z})$ denote the acceleration and velocity of UAV quadcopter in the inertial frame, respectively. $(\ddot{\theta}, \ddot{\varphi}, \ddot{\psi})$ and $(\dot{\theta}, \dot{\varphi}, \dot{\psi})$ denote angular acceleration and angular velocity of the Euler angles (roll, pitch, yaw) respectively. $\mathrm{C}$ and $\mathrm{S}$ denote Cos and Sin, respectively.

The numerical simulation drone quadcopter parameters of the Eq. (1) to Eq. (7) are taken from [5] as shown in Table 1.

\section{Multi-PID-PSO controller design}

The proposed on-line multi-PID-PSO controller is used in this work to solve the highly coupled between the inputs and the outputs states of the UAV quadrotor and the nonlinear dynamic behaviour with under-actuated system through generating precisely and quickly the optimal four rotor speeds action. As well as to follow the desired Spiral and Cyclone UAV quadcopter paths and to achieve the UAV quadrotor is stabilized during flying with minimum position error, without oscillation and minimum overshoot in the altitude of quadrotor system. The platform of the proposed multi-PID-PSO path following controller is shown in Fig. 2.

The proposed controller consists of two steps:

First Step: In this work, it is used six PID controllers because the quadcopter drone has $6 \mathrm{DOF}$ and it has 6 states as Eq. (1) to Eq. (6). There are six discrete PID control law equations are represented in Eq. (8) as follows [21, 22]: 


$$
\begin{aligned}
u_{\alpha}(k)=k_{p \alpha}( & k) e_{\alpha}(k) \\
& +k_{i \alpha}(k)\left(e_{\alpha}(k)\right. \\
& \left.+e_{\alpha}(k-1)\right) \\
& +k_{d \alpha}(k)\left(e_{\alpha}(k)\right. \\
& \left.-e_{\alpha}(k-1)\right) \\
& +u_{\alpha}(k-1)
\end{aligned}
$$

Where, $\alpha$ denotes to the different output state of the quadcopter drone that there are $(\mathrm{x}, \mathrm{y}, \mathrm{z})$ and $(\varphi, \theta$, $\psi) . k_{p}$ denotes the proportional gain; $k_{i}$ denotes the integral gain; $k_{d}$ denotes the derivative gain; $u(k)$ denotes the control action and $e(k)$ denotes the error signal.

However, the control input vector $\mathrm{U}$ can be expressed as a function of rotation speeds $\left(w_{1}, w_{2}, w_{3}\right.$, $w_{4}$ ) in order to control the altitude and attitude of UAV quadrotor as follows $[1,5]$ :

$$
\begin{gathered}
U_{1}=b\left(w_{1}^{2}+w_{2}^{2}+w_{3}^{2}+w_{4}^{2}\right) \\
U_{2}=b\left(w_{4}^{2}-w_{2}^{2}\right) \\
U_{3}=b\left(w_{3}^{2}-w_{1}^{2}\right) \\
U_{4}=d\left(w_{1}^{2}-w_{2}^{2}+w_{3}^{2}-w_{4}^{2}\right)
\end{gathered}
$$

Second Step: In this work, the control gain $k_{p \alpha}, k_{i \alpha}$ and $k_{d \alpha}$ (eighteen) parameters in each particle which are learned and tuned using the on-line PSO algorithm in order to speed up the transient state of the six states of the UAV quadcopter by changing the rotation speeds control actions that leads the UAV quadcopter to follow the desired path quickly with minimum location and rotation errors, without over

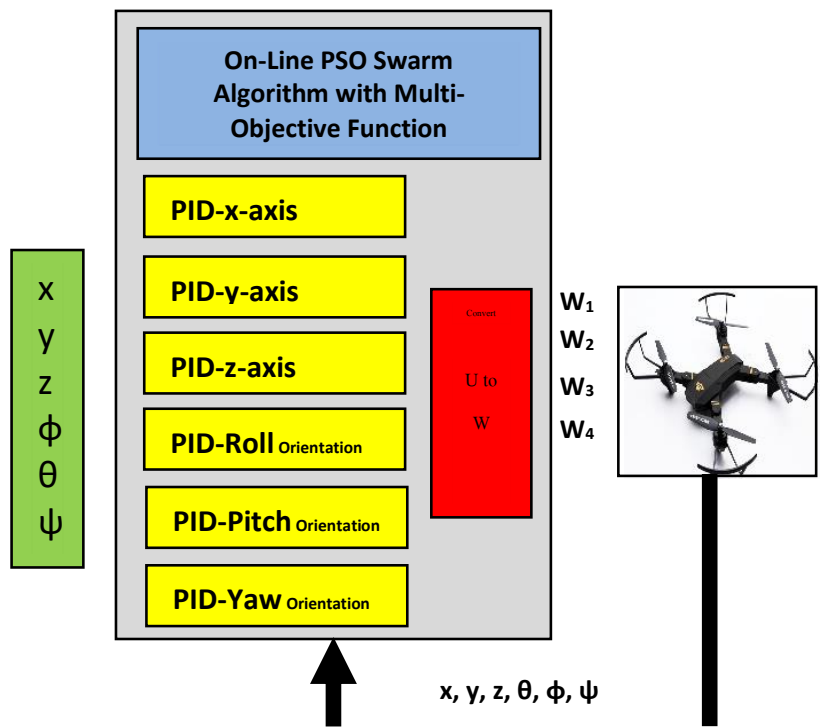

Figure. 2 The platform of multi-PID-PSO controller design shoot, and with no oscillation in the output states responses. The particle's "the control gains $k_{p \alpha}, k_{i \alpha}$ and $k_{d \alpha}$ parameters" velocity update using Eq. (13) and to represents the particle's position update using Eq. (14) as follows [23, 24]:

$$
\begin{aligned}
v_{i}(k+1)=w & \cdot v_{i}(k) \\
& +c_{1} r_{1}\left[L_{\text {best }-i}-x_{i}(k)\right] \\
& +c_{2} r_{2}\left[G_{\text {best }}-x_{i}(k)\right] \\
x_{i}(k+1)= & x_{i}(k)+v_{i}(k+1)
\end{aligned}
$$

Where, $c_{1}$ and $c_{2}$ are cognitive coefficients and there are equal to 1.25 . The $r_{1}$ and $r_{2}$ are two uniform random numbers from (0 to 1$) ; i^{\text {th }}$ denotes the number of particles, $x_{i}$ denotes the particle the control gain $k_{p \alpha}, k_{i \alpha}$ and $k_{d \alpha}$ parameters; $\mathrm{G}_{\text {best }}$ is Global best position, and $\mathrm{L}_{\text {best }}$ is the Local best position, $\mathrm{V}_{i}$ is the velocity which is adjusted at each time step that is equal to 0.01 second.

The proposed multi-objective function in this work is mean square error function that is used to minimize the location error, rotation error and translation speed coordination error between the desired and actual output of the UAV quadcopter by using Eq. (15), (16) and Eq. (17).

$$
\begin{aligned}
& e_{\alpha}(k+1)=D_{\alpha}(k+1)-O_{\alpha}(\mathrm{k}+1) \\
& \operatorname{Spe}_{\alpha}(k+1)=D \operatorname{Sp}_{\alpha}(k+1) \\
& -O S p_{\alpha}(\mathrm{k}+1) \\
& E=\frac{1}{K} \sum_{j}^{K} \sum \frac{1}{\beta} \sum_{i i=1}^{\beta}\left(e_{\alpha}^{i i}(k+1)\right)^{2} \\
& +\left(\operatorname{Spe}_{\alpha}^{i i}(k+1)\right)^{2}
\end{aligned}
$$

Where, $\beta$ denotes the number of iterations; $k^{\text {th }}$ denotes the number of samples; $e_{\alpha}^{i i}$ : is the location and rotation errors of each iteration. $O_{\alpha}^{i i}$ : is the actual output of the quadcopter of each iteration. $D_{\alpha}^{i i}$ : is the desired output of the location and rotation at each iteration for the drone quadcopter. $S p e_{\alpha}^{i i}$ : is the translation coordinate speed errors of each iteration. $O S p_{\alpha}^{i i}:$ is the actual translation coordinate speed output of the quadcopter of each iteration. $D S p_{\alpha}^{i i}$ : is the desired output of the translation coordinate speed at each iteration for the drone quadcopter.

\section{Numerical simulation results}

The UAV quadrotor model is shown in Fig. 1 which is considered as under-actuated quadrotor system with four inputs and six states outputs with 
highly coupled nonlinear dynamics behaviour. MATLAB simulation package is used to apply the proposed on-line tuning control gains algorithm for multi-PID-PSO structure of the controller by carrying out the $4^{\text {th }}$ order Range Kutta method and solving Eq. (1) to Eq. (6) with 0.01 second sampling time in order to solve the problem definition of the UAV quadrotor as well as to follow the desired UAV quadcopter path and to achieve the UAV quadrotor is stabilized during flying with minimum position error, without oscillation and minimum overshoot in the altitude of quadrotor system. To investigate the efficiency of the proposed controller at strongly dynamics Spiral and Cyclone paths tracking and to confirm the UAV quadrotor in both desired (location and rotation) are stabilized, we put the UAV quadrotor in two case studies as follows:

\section{Case I}

The spiral and turns on itself desired path during 35 second as follows:

$$
X_{d}=4 \sin \left(\frac{2 \pi t}{15}\right)
$$

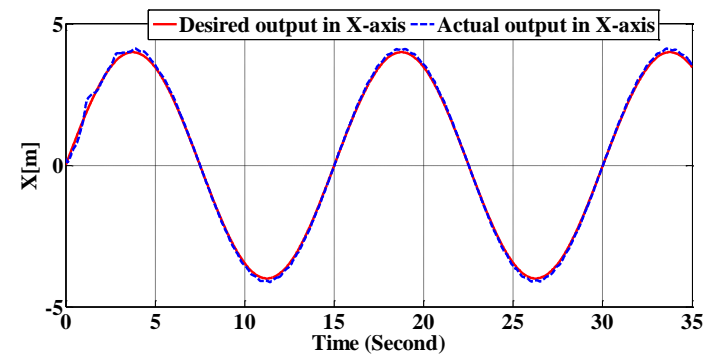

(a)

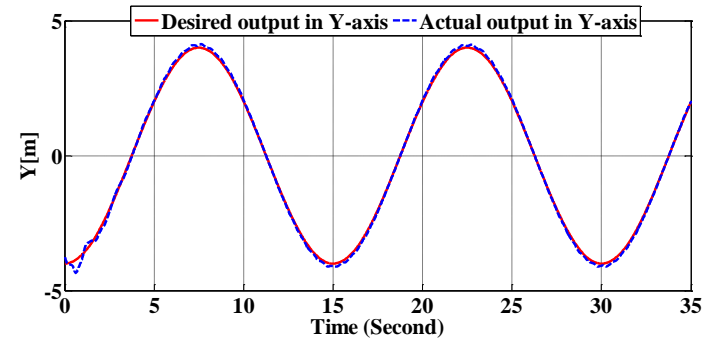

(b)

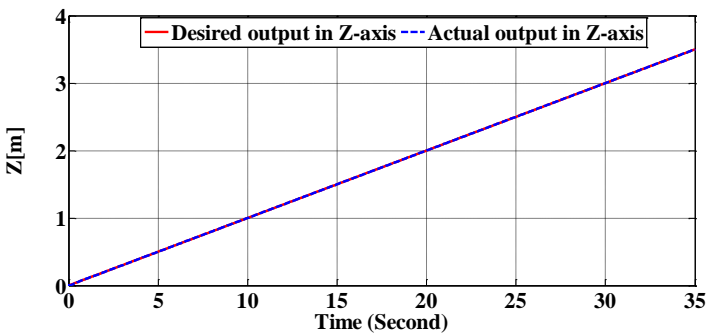

(c)

Figure. 3 The spiral desired path with translation coordinates: (a) $\mathrm{x}$ axis, (b) y axis and (c) $\mathrm{z}$ axis

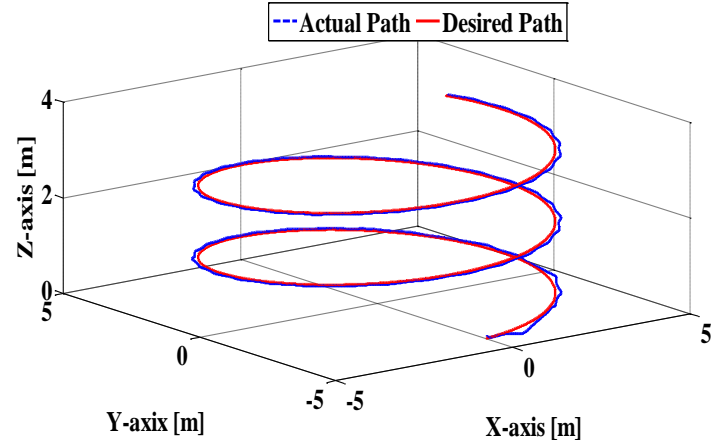

Figure. 4 The 3D simulation spiral path tracking for the UAV quadrotor

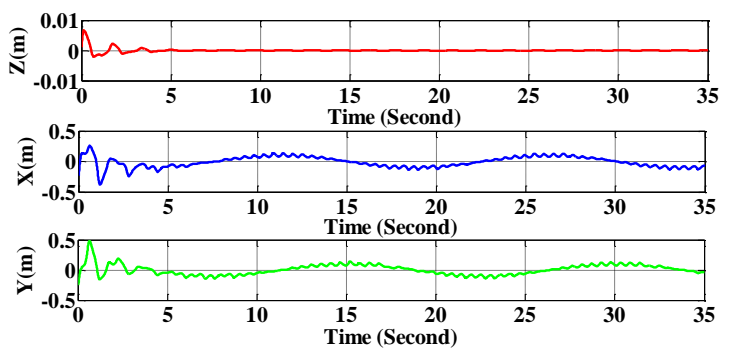

Figure. 5 The translation error coordinates in $(\mathrm{z}, \mathrm{x}, \mathrm{y})$

$$
\begin{gathered}
Y_{d}=-4 \cos \left(\frac{2 \pi t}{15}\right) \\
Z_{d}=0.1 t \\
\psi_{d}=\pi \sin \left(\frac{2 \pi t}{15}\right)
\end{gathered}
$$

The initial position of the UAV quadrotor is $\left(\mathrm{x}_{\mathrm{o}}\right.$, $\left.\mathrm{y}_{\mathrm{o}}, \mathrm{z}_{\mathrm{o}}\right)=(0.25,-3.75,0) \mathrm{m}$ respectively.

Fig.3 shows the desired path with translation coordinates $(\mathrm{x}, \mathrm{y}, \mathrm{z})$ and to show the 3D simulation path tracking for the UAV quadrotor as in Fig. 4 that has a fast flying with small oscillation in the starting on the following the desired path only.

Fig. 5 shows the small translation error coordinates and did not exceed $\pm 5 \mathrm{~cm}$ and the overshoot of the altitude is less than $1 \mathrm{~cm}$. In Fig. 6, the orientation yaw error coordination did not exceed $0.04 \mathrm{rad}$ that means the proposed controller behaviour precision.

Based on the proposed output of the PID x-axis in Eq. (22) and the output of the PID y-axis in Eq. (23), we are obtained the desired Roll and Pitch orientations as shown in Fig. 7.

$$
\begin{aligned}
\varphi_{d} & =\frac{M}{U_{1}}\left(u_{x} \operatorname{Sin}(\psi)-u_{y} \operatorname{Cos}(\psi)\right) \\
\theta_{d} & =\frac{M}{U_{1}}\left(u_{x} \operatorname{Cos}(\psi)+u_{y} \operatorname{Sin}(\psi)\right)
\end{aligned}
$$




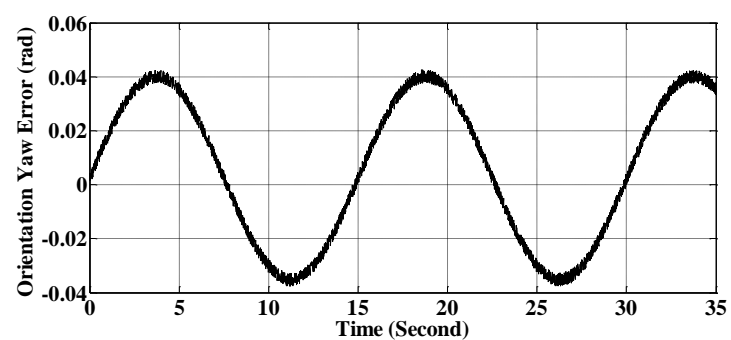

Figure. 6 The orientation yaw error coordination

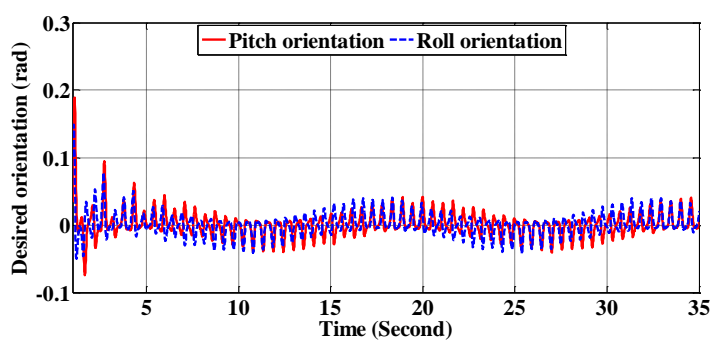

Figure. 7 The desired Roll and Pitch orientations

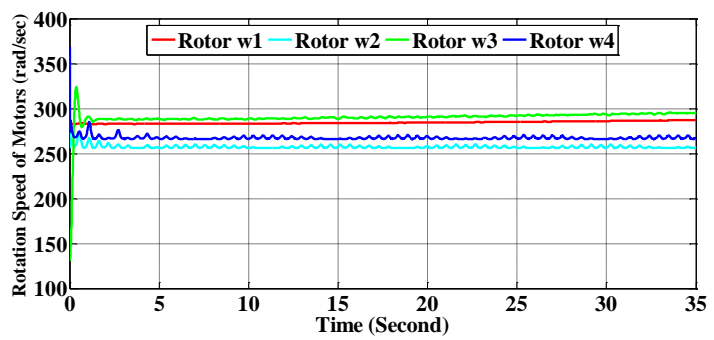

Figure. 8 The response of the rotation speed of each rotor in the UAV quadrotor

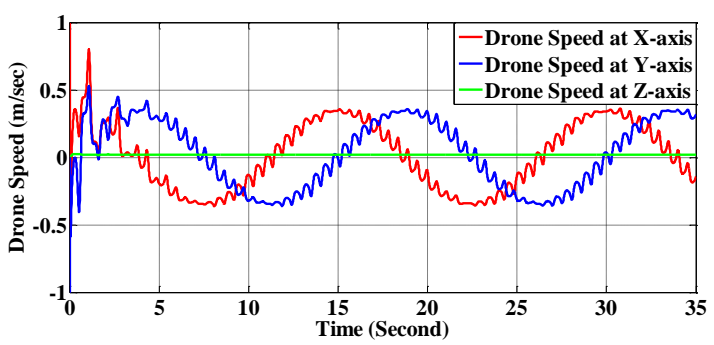

Figure. 9 The velocities of UAV quadcopter in the inertial frame

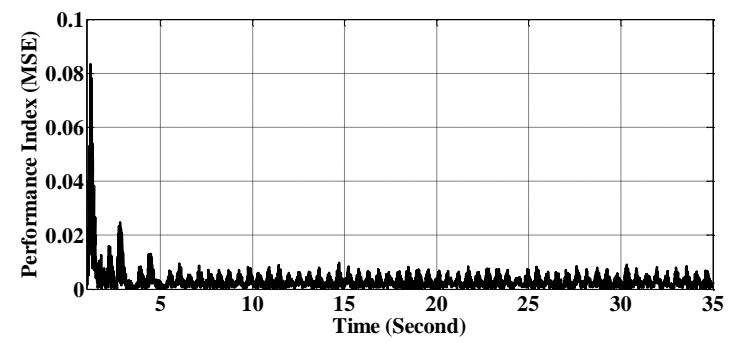

Figure. 10 The multi-objective function performance
Table 2. The search area of on-line PSO control gains parameters values.

\begin{tabular}{|c|c|c|c|}
\hline $\begin{array}{c}\text { Controller } \\
\text { Types }\end{array}$ & $\begin{array}{c}\text { Rang of } k_{p} \\
\text { Values }\end{array}$ & $\begin{array}{c}\text { Range of } \mathbf{k}_{\mathbf{i}} \\
\text { Values }\end{array}$ & $\begin{array}{c}\text { Range of kd } \\
\text { Values }\end{array}$ \\
\hline PID-x-axis & \multirow{2}{*}{ [9.8 to 0.22 ] } & \multirow{2}{*}{ [2.1 to 0.011$]$} & \multirow{2}{*}{ [3.1 to 0.17$]$} \\
\hline PID-y-axis & & & \\
\hline PID-z-axis & [7.8 to 4.3$]$ & [1.6 to 1.2$]$ & [0.77 to 0.23$]$ \\
\hline $\begin{array}{l}\text { PID-Roll } \\
\text { Orientation }\end{array}$ & \multirow{2}{*}{ [1.9 to 1.1$]$} & \multirow{2}{*}{ [1.9 to 0.22 ] } & \multirow{2}{*}{ [2.1 to 0.99$]$} \\
\hline $\begin{array}{l}\text { PID-Pitch } \\
\text { Orientation }\end{array}$ & & & \\
\hline $\begin{array}{l}\text { PID-Yaw } \\
\text { Orientation }\end{array}$ & [0.15 to 0.1$]$ & {$[0.2$ to 0.1$]$} & [0.2 to 0.1$]$ \\
\hline
\end{tabular}

Fig. 8 shows the response of the rotation speeds of each rotor in the UAV quadrotor where the response is fast action with small oscillation and no saturation state as well as no spikes actions because the effectiveness of the on-line tuning control gains that used in the proposed multi-PID-PSO controller with multi-objective function are generated smooth and stable values of the multi-PID-PSO controller's parameters. Moreover, these control actions have the ability to smoothly track the Spiral desired location and rotation of the UAV quadrotor.

Fig. 9 shows the response of the UAV speed in translation coordinate $(\mathrm{x}, \mathrm{y}, \mathrm{z})$ during flight in the Spiral path.

The multi-objective function performance of the on-line tuning control gains algorithm is shown in Fig. 10 that convergence the location, rotation and translation speed coordination errors throughout running 35 second when it is used number of iteration is equal to 50 .

Table 2 shows the proposed search area of on-line PSO control gains parameters values that there are used in the proposed multi-PID-PSO controller in order to stabilize and speed up the UAV quadrotor flying in the transient response of the closed loops control drone system.

Case II

The Cyclone desired path for UAV quadrotor is carried out during 65 second as follows:

The UAV quadrotor ups to $1.3 \mathrm{~m}$ of altitude during 13 second where the desired path with translation coordinates $\left(X_{d}=0, Y_{d}=0\right)$ and $Z_{d}=$ $0.1 t$

So, the initial position of the UAV quadrotor is $\left(\mathrm{x}_{0}, \mathrm{y}_{\mathrm{o}}, \mathrm{z}_{\mathrm{o}}\right)=(0,0,1.3) \mathrm{m}$ respectively, before the UAV quadrotor makes Cyclone trajectory between the time (13 to 65) second.

$$
X_{d}=0.1\left(t_{65}-t_{13}\right) \sin \left(\frac{2 \pi\left(t_{65}-t_{13}\right)}{15}\right)
$$




$$
\begin{gathered}
Y_{d} \\
=-0.1\left(t_{65}-t_{13}\right) \cos \left(\frac{2 \pi\left(t_{65}-t_{13}\right)}{15}\right) \\
Z_{d}=0.1 t \\
\psi_{d}=\pi \sin \left(\frac{2 \pi\left(t_{65}-t_{13}\right)}{15}\right)
\end{gathered}
$$

Fig.11 shows the Cyclone desired path with translation coordinates $(x, y, z)$ and to show the $3 \mathrm{D}$ simulation path tracking for the UAV quadrotor as in Fig. 12 that has a fast flying with small oscillation during tracking the desired path.

In Fig. 13 the translation error coordinates is less than $\pm 5 \mathrm{~cm}$ and the overshoot value of the altitude during 13 second did not exceed $1 \mathrm{~cm}$ that means the percentage overshoot did not exceed 1\%. Fig. 14 shows the maximum value of orientation yaw error coordination is less than 0.04 rad.

Fig. 15 shows the rotation speeds responses of each rotor in the UAV quadrotor during smoothly tracking the Cyclone path. The responses are fast

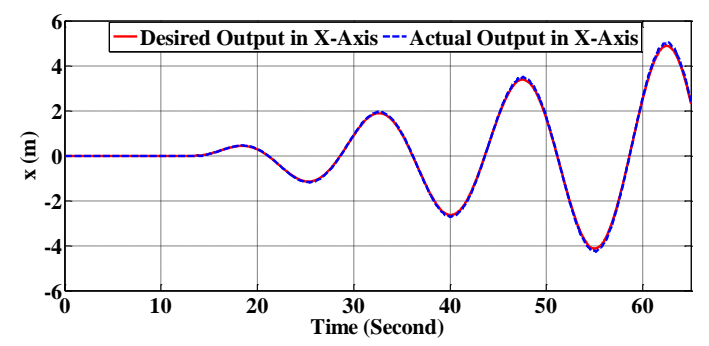

(a)

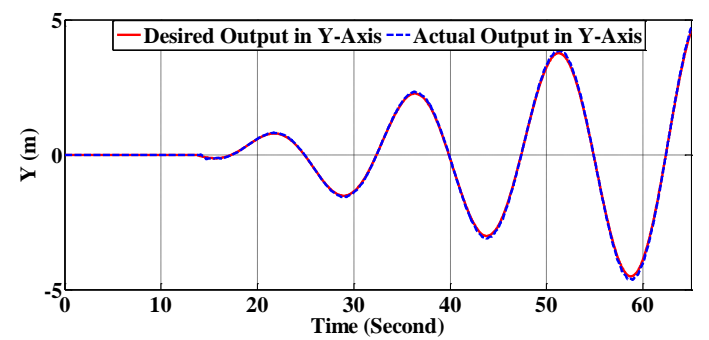

(b)

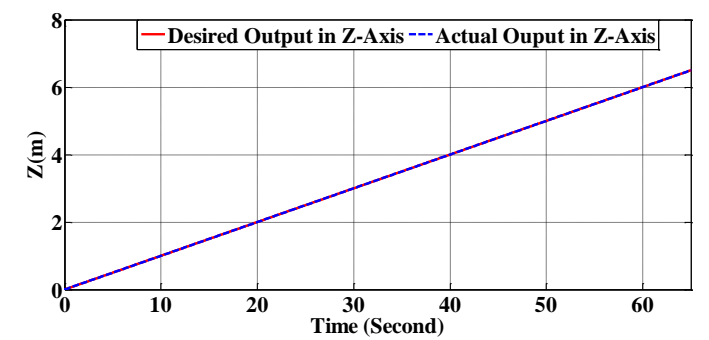

(c)

Figure. 11 The Cyclone desired path with translation coordinates: (a) $\mathrm{x}$ axis, (b) y axis and (c) $\mathrm{z}$ axis

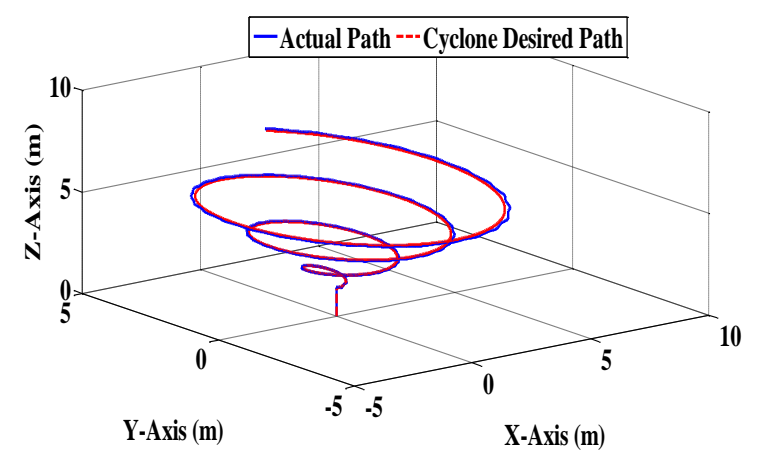

Figure. 12 The 3D simulation Cyclone path tracking for the UAV quadrotor

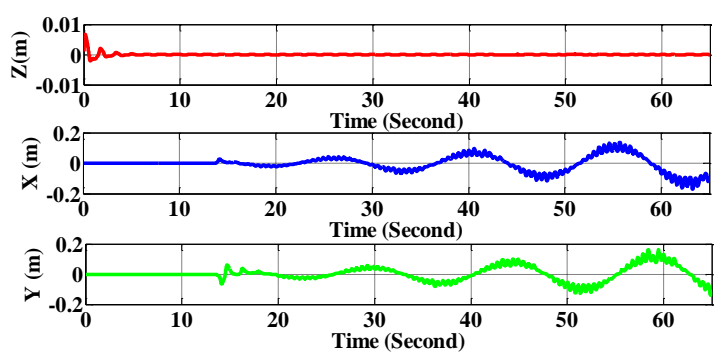

Figure. 13 The translation error coordinates in (z, x, y).

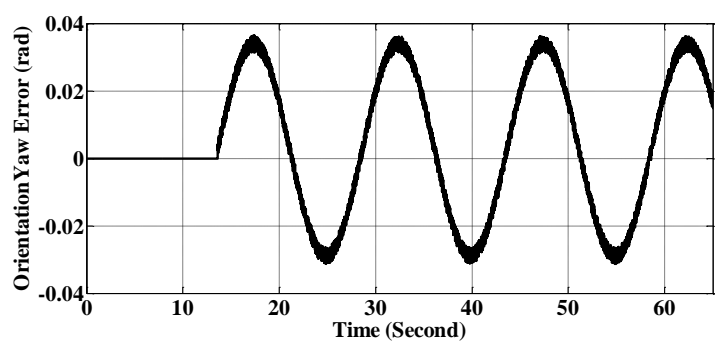

Figure. 14 The orientation yaw error coordination

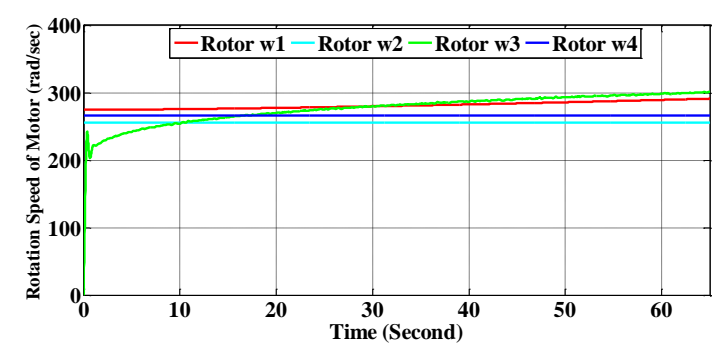

Figure. 15 The response of the rotation speed of each rotors in the UAV quadrotor

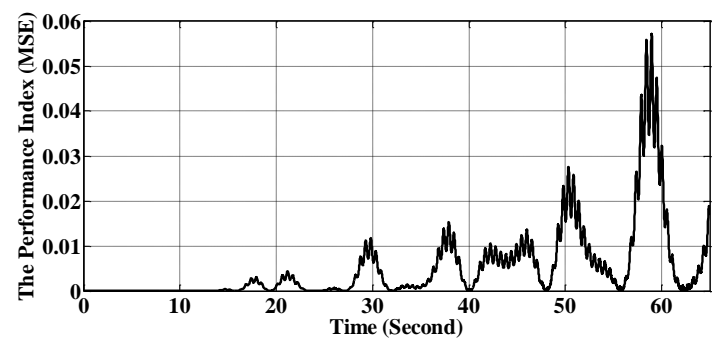

Figure. 16 On-line multi-objective function performance. 
Table 3. Comparison simulation results between the proposed controller and other types of PID controller

\begin{tabular}{|c|c|c|c|}
\hline $\begin{array}{c}\text { Type of } \\
\text { Controller }\end{array}$ & $\begin{array}{c}\text { Type of } \\
\text { Performance } \\
\text { Index }\end{array}$ & Overshoot & $\begin{array}{c}\text { Steady- } \\
\text { State } \\
\text { Error }\end{array}$ \\
\hline $\begin{array}{c}\text { PID with } \\
\text { try and } \\
\text { error } \\
\text { tuning } \\
\text { algorithm } \\
\text { [1] }\end{array}$ & $\begin{array}{c}\text { Off-Line } \\
\text { ISE=4.72 }\end{array}$ & $4.08 \%$ & $30 \mathrm{~cm}$ \\
\hline $\begin{array}{c}\text { PID with } \\
\text { genetic } \\
\text { tuning } \\
\text { algorithm } \\
\text { [5] }\end{array}$ & $\begin{array}{c}\text { Off-Line } \\
\text { ISE } 2.14\end{array}$ & $1 \%$ & $10 \mathrm{~cm}$ \\
\hline $\begin{array}{c}\text { Proposed } \\
\text { controller }\end{array}$ & $\begin{array}{c}\text { On-Line } \\
\text { ISE }=1.08\end{array}$ & $1 \%$ & $5 \mathrm{~cm}$ \\
\hline
\end{tabular}

action with small oscillation and no saturation state as well as no spikes actions because of the effectiveness of the on-line tuning control gains that are used in the proposed multi-PID-PSO controller with multi-objective function that are generated by a smooth and stable values of the multi-PID-PSO controller's parameters.

The multi-objective function performance of the on-line tuning control gains algorithm is shown in Fig. 16 that working to minimize the location error and the translation speed coordination error during the radius of the Cyclone path increased throughout running 65 second and fifty iterations are used in each sample for function evaluation.

To confirm the effectiveness of this work, we are compared the simulation results of the proposed controller with other types of PID controller results that are taken from $[1,5]$ as shown in Table 3 .

\section{Conclusions}

In this paper, an on-line multi-PID-PSO controller has been designed and simulated for flying UAV quadrotor system and tracking Spiral and Cyclone paths using MATLAB simulation package. The UAV quadrotor has highly coupled and nonlinear system, therefore, the proposed controller has excellent ability for solving the problem statement of the UAV model in term of the following:

Best value of speed rotors control actions that were generated for decoupling system states and nonlinear behaviour of the UAV quadrotor system.

The UAV quadrotor is fast flied and reached the Spiral and Cyclone paths with slight oscillation.

The span tracking translation error of the location reached approximately $\pm 5 \mathrm{~cm}$.

Smooth speed actions without spikes and no saturation state led to follow precision of the desired strongly dynamics Spiral and Cyclone paths because multi-objective function is used with on-line tuning control gains and stable positive values are obtained by PSO algorithm.

So, the experimental implementation of an online multi-PID-PSO controller schematic with UAV model will be addressed in the future work.

\section{Conflicts of Interest}

The authors declare no conflict of interest.

\section{Author Contributions}

Mohamed Najm Abdullah and Khulood Eskander Dagher developed multi-PID-PSO controller for UAV model and verified the proposed controller. Mohamed Najm Abdullah explained the swarm optimization algorithm (PSO). Khulood Eskander Dagher presented the dynamics UAV model. Both authors discussed the numerical simulation results contributed to the final work.

\section{References}

[1] J. Chaoraingern, V. Tipsuwanporn, and A. Numsomran, "Modified Adaptive Sliding Mode Control for Trajectory Tracking of Mini-drone Quadcopter Unmanned Aerial Vehicle", International Journal of Intelligent Engineering and Systems, Vol.13, No.5, pp.145-158, 2020.

[2] B. Selma, S. Chouraqui, and H. Abouaissa, "Fuzzy Swarm Trajectory Tracking Control of Unmanned Aerial Vehicle", Journal of Computational Design and Engineering, Vol.7, No.4, pp.435-447, 2020.

[3] C. Huang, "Design of Decoupling Fuzzy Logic Controller for Quadrotor UAV", Journal of Physics, Vol. 1684, pp. 1-9, 2020.

[4] D. Li, "Fuzzy PID Controller to Control the Attitude of Quadrotor UAV", Gyancity Journal of Engineering and Technology, Vol. 6, No. 1, pp. 1-11, 2020.

[5] S-E-I. Hasseni, L. Abdou, and H-E. Glida, "Parameters Tuning of a Quadrotor PID Controllers by using Nature-Inspired Algorithms", Evolutionary Intelligence, doi.org/10.1007/s12065-019-00312-8, 2019.

[6] R. Guardeno, M. j. Lopez, and V. M. Sanchez, "MIMO PID Controller Tuning Method for Quadrotor based on LOR/LOG Theory", Robotics, Vol. 8, No. 36, pp. 2-28, 2019.

[7] M. A. Khodja, M. Tadjine, M. S. Boucherit, and M. Benzaoui, "Tuning PID Attitude Stabilization of a Quadrotor using Particle Swarm Optimization (Experimental)", 
International Journal for Simulation and Multidisciplinary Design Optimization, Vol. 8, No. A8, pp. 1-9, 2017.

[8] X. Chen, and L. Wang, "Quadrotor Cascade PID Controller Automatic Tuning", In: Proc. of International Conf. On Australian Control, Newcastle, Australia, pp. 311-316, 2016.

[9] Z. He, and L. Zhao, "A Simple Attitude Control of Quadrotor Helicopter based on ZieglerNichols Rules for Tuning PD Parameters", The Scientific World Journal, Vol. 2014, pp. 1-13, 2014.

[10] S. Zeghlache, M. Z. Ghellab, and A. Bouguerra, "Adaptive Type-2 Fuzzy Sliding Mode Control using Supervisory Type-2 Fuzzy Control for 6 DOF Octo-rotor Aircraft", International Journal of Intelligent Engineering and Systems, Vol. 10, No. 3, pp. 47-57, 2017.

[11] A. Bouguerra, D. Saigaa, K. Kara, and S. Zeghlache, "Fault-Tolerant Lyapunov-GainScheduled PID Control of a Quadrotor UAV", International Journal of Intelligent Engineering and Systems, Vol. 8, No. 2, pp. 1-6, 2015.

[12] H. Maqsood, and Y. Ou, "Nonlinear Disturbance Observer based Sliding Mode Control of Quadrotor Helicopter", Journal of Electrical Engineering and Technology, Vol. 15, pp. 1453-14-61, 2020.

[13] H. E. Glida, L. Abdou, A. Chelihi, C. Sentouh, and S-E-I. Husseni, "Optimal Model-Free Backstepping Contrl for a Quadrotor Helicopter", Nonlinear Dynamics, Vol. 100, pp. 3449-3468, 2020.

[14] K. U. Lee, Y. H. Choi, and J. B. Park, "Backstepping based Formation Control of Quadrotors with the State Transformation Technique", Applied Sciences, Vol. 7, No. 1170, pp.1-25, 2017.

[15] P. Ru, and K. Subbarao, "Nonlinear Model Predictive Control for Unmanned Aerial Vehicles", Aerospace, Vol. 4, No. 31, pp.1-26, 2017.

[16] P. N. Chikasha, and C. Dube, "Adaptive Model Predictive Control of a Quadrotor", In: Proc. of International Conf. On IFAC Paper, Vol. 50, No. 2, pp. 157-162, 2017.

[17] J. Muliadi, and B. Kusumoputro, "Neural Network Control System of UAV Altitude Dynamics and Its Comparison with the PID Control System", Journal of Advanced Transportation, Vol. 2, No. 2, pp. 1-18, 2018.

[18] O. Cakir, and T. Yuksel, "Neural Network Control for Quadrotors", American Scientific Research Journal for Engineering, Technology, and Sciences, Vol. 31, No. 1, pp. 191-200, 2017.
[19] M. Lower, and W. Tarnawski, "Quadrotor Navigation using the PID and Neural Network Controller", Theory and Engineering of Complex Systems and Dependability, Vol. 365, pp. 265-274, 2015.

[20] H. T. Nguyen, T. V. Quyen, C. V. Nguyen, A. M. Le, H. T. Tran, and M. T. Nguyen, "Control Algorithms for UAVs: A Comprehensive Survey", EAI Endorsed Transactions on Industrial Networks and Intelligent Systems, Vol. 7, No. 23, pp. 1-11, 2020.

[21] A. Sento and Y. Kitjaidure, "A Neural Network PID-Like Controller using a Hybrid of Online Actor-Critic Reinforcement Algorithm with the Square Root Cubature Kalman Filter", International Journal of Intelligent Engineering and Systems, Vol. 11, No. 6, pp. 261-270, 2018.

[22] A. Al-Araji, "Genetic Algorithm and Elman Neural Network Used for Tuning the Parameters of the PID Neural Controller based Model Reference". Iraqi Journal of Computer, Communication, Control and Systems Engineering, Vol. 5, No. 1, pp. 111-126, 2005.

[23] R. H. Al-Rubayi, M. K. Abd, and F. M. Flaih, "A New Enhancement on PSO Algorithm for Combined Economic-Emission Load Dispatch Issues", International Journal of Intelligent Engineering and Systems, Vol. 13, No. 1, pp. 77-85, 2020.

[24] A. Al-Araji, “Applying Cognitive Methodology in Designing On-Line Auto-Tuning Robust PID Controller for the Real Heating System". Journal of Engineering, Vol. 20, No. 9, pp. 4361, 2014. 